\title{
Serviço Nacional de Saúde: Dos Desafios da Atualidade às Transformações Necessárias
}

\section{National Health Service: Responding to Current Challenges Through Necessary Transformations}

\author{
Constantino SAKELLARIDES $\square^{1}$ \\ Acta Med Port 2020 Feb;33(2):133-142 - https://doi.org/10.20344/amp.12626
}

\begin{abstract}
RESUMO
Após uma década de evidentes dificuldades, o Serviço Nacional de Saúde necessita de um significativo investimento público para o seu desenvolvimento. Isso significa repor e acrescentar recursos ao Serviço Nacional de Saúde. Mas quer dizer também adquirir as capacidades necessárias à gestão da sua transformação. Os desafios de saúde não são hoje os mesmos de há 40 anos: (i) Observa-se um considerável aumento da prevalência das pessoas com morbilidade múltipla, de evolução prolongada, requerendo uma utilização contínua de serviços de saúde; (ii) É necessário atrasar e atenuar a expressão da morbilidade múltipla; (iii) Neste contexto, é particularmente importante uma forte aposta na integração dos cuidados, gerindo o percurso das pessoas através do serviços de que necessitam. As transformações indispensáveis para responder a estes desafios são complexas e muito exigentes: (i) Há que reconhecer que uma gestão efetiva das necessárias 'mudanças adaptativas de proximidade', requer um elaborado enquadramento analítico, estratégico, de boa governança e governação, que ainda não temos; (ii) É preciso ativar, no conjunto da sociedade portuguesa, fatores indutores e facilitadores de mudança - arquiteturas, processo e instrumentos colaborativos; (iii) Uma forte consciência coletiva da natureza dos desafios de saúde da atualidade e uma acrescida capacidade em gerir mudanças socialmente complexas, permitirá organizar uma direção estratégica para o Serviço Nacional de Saúde, capaz de atuar efetivamente em domínios críticos para o seu desenvolvimento - financiamento, gestão de infraestruturas e recursos, desenvolvimento organizacional no âmbito da administração pública portuguesa, uma política para as profissões de saúde e a articulação com os setores social e privado. Sem um investimento imediato e significativo na capacidade de gerir as mudanças necessárias, as dificuldades do Serviço Nacional de Saúde em corresponder a aquilo que os portugueses precisam, não deixarão de se acentuar nos próximos anos.
\end{abstract}

Palavras-chave: Disparidades em Assistência à Saúde; Indicadores Básicos de Saúde; Política de Saúde; Portugal; Programas Nacionais de Saúde

\section{ABSTRACT}

After a decade experiencing clear difficulties, the Portuguese National Health Service needs a significant public investment for its development. This means more resources for the National Health Service, but also an increased capacity to manage its necessary transformation. Current health challenges differ significantly from those prevailing when the National Health Service was institutionalized, 40 years ago: (i) A significant increase in the prevalence of patients with multimorbidity has been observed; (ii)There is a need to delay and attenuate the expression of multimorbidity; (iii) In this context, a strong investment in integrating health care is of a paramount importance. The transformations that the National Health Service needs to respond to these challenges are very complex and demanding: (i) Effective local adaptive change management requires a very elaborate strategic, governance and government framework, which is yet to be developed; (ii) Collaborative architectures, processes and tools need to be promoted in order to induce and facilitate change in the Portuguese health system; (iii) Common understanding of current challenges and increased capabilities to deal with complex change processes may facilitate the implementation of effective strategic management in the National Health Service, focused on its more critical domains - financing, infrastructures and resource management, organizational development, policies for attracting and retaining health professional and cooperation with the social and private sectors. Such a transformation agenda is urgently needed. Keywords: Health Policy; Health Status Indicators; Healthcare Disparities; National Health Programs; Portugal

\section{INTRODUÇÃO}

As políticas de saúde têm como objetivo fazer evoluir os sistemas de saúde no sentido de melhor responder àquilo que as pessoas precisam em termos de promoção e proteção da saúde - dar resposta aos desafios de saúde da atualidade.

Após a crise financeira e económica, desencadeada na segunda metade da última década, assistiu-se a uma clara hierarquização das políticas públicas: primeiro as politicas financeiras, depois as preocupações de natureza económica, ficando as de caráter social, como as relativas à saúde, com o que resta das disponibilidades orçamentais do Estado.1,2

Isso levou à rápida degradação dos serviços públicos,

particularmente do Serviço Nacional de Saúde (SNS), e das condições de trabalho de quem lá exerce a sua profissão. Os seus efeitos fizeram-se sentir, não só imediatamente, mas também, cumulativamente mais a médio prazo. São hoje ainda muito aparentes. Um destes efeitos foi centrar o debate do SNS na questão do seu financiamento.

É necessário passar da hierarquização das políticas públicas para a sua harmonização: conciliar os objetivos de bem-estar das pessoas, com os do crescimento da economia, com os das contas públicas seguras.

A contribuição da saúde e do SNS para a harmonização das políticas públicas deve começar pela explicitação de objetivos de saúde e de desenvolvimento do SNS que

1. Professor Catedrático jubilado. Escola Nacional de Saúde Pública. Universidade NOVA de Lisboa. Lisboa. Portugal.

$\square$ Autor correspondente: Constantino Sakellarides. sakellaridessak@ensp.unl.pt

Recebido: 26 de julho de 2019 - Aceite: 31 de julho de 2019 | Copyright @ Ordem dos Médicos 2020 
decorrem dos desafios de que os portugueses hoje enfrentam neste domínio.

Há 40 anos o SNS propôs-se responder às necessidades de um país jovem, que não oferecia a muitos dos seus cidadãos os serviços de saúde que precisavam, nem sequer uma resposta a tempo aos episódios comuns da doença aguda.

No decurso das últimas quatro décadas o país mudou. Cresceu economicamente, envelheceu e tornou-se mais exigente.

É importante repor e acrescentar recursos ao SNS. Mas parece igualmente evidente que é preciso fazê-lo, transformando-o simultaneamente, em função das necessidades atuais dos portugueses.

Assim, é intenção deste trabalho proporcionar um resumo atualizado e documentado sobre o presente e o futuro imediato do SNS, organizado em duas dimensões complementares:

A primeira diz respeito à identificação dos principais desafios de saúde na atualidade;

A segunda refere-se à gestão de uma mudança de elevada complexidade, necessária para responder efetivamente àqueles desafios.

\section{PARTE I. Desafios atuais}

É indispensável resumir de uma forma tecnicamente sustentada e politicamente eficaz os principais desafios de saúde na atualidade. Tornar claro aquilo que as pessoas realmente precisam, é já em si uma importante contribuição para promover as mudanças necessárias.
São, essencialmente, três estes grandes desafios:

- Primeiro: compreender a importância, para a saúde e para os cuidados de saúde, da alta prevalência de pessoas com múltiplos problemas de saúde "morbilidade múltipla" (MM) - de natureza diversa, persistentes, com uma evolução prolongada e que requerem uma utilização frequente de cuidados de saúde;

- Segundo: atuar no sentido de atrasar o início e atenuar a expressão da MM nas pessoas, prestando a atenção devida ao desenvolvimento infantil, à promoção de uma "vida ativa" e à gestão dos riscos de doença no futuro, sem desvalorizar a importância da promoção da saúde, em si própria;

- Terceiro: responder adequadamente às necessidades das pessoas com MM, superando a fragmentação, descoordenação e descontinuidade dos cuidados de saúde e promovendo a integração desses cuidados, através da boa gestão do percurso das pessoas pelos serviços de saúde de que necessitam.

\section{Desafio um: pessoas com morbilidade múltipla}

A questão da morbilidade múltipla tem sido referida na literatura médica portuguesa, principalmente a partir da década passada, por vários autores. ${ }^{2-9}$

O estudo de referência sobre a epidemiologia da MM, que teve lugar na Escócia, com base nos registos clínicos de cerca de um milhão e 700 mil pessoas, provenientes de 314 unidades prestadoras de cuidados de saúde,

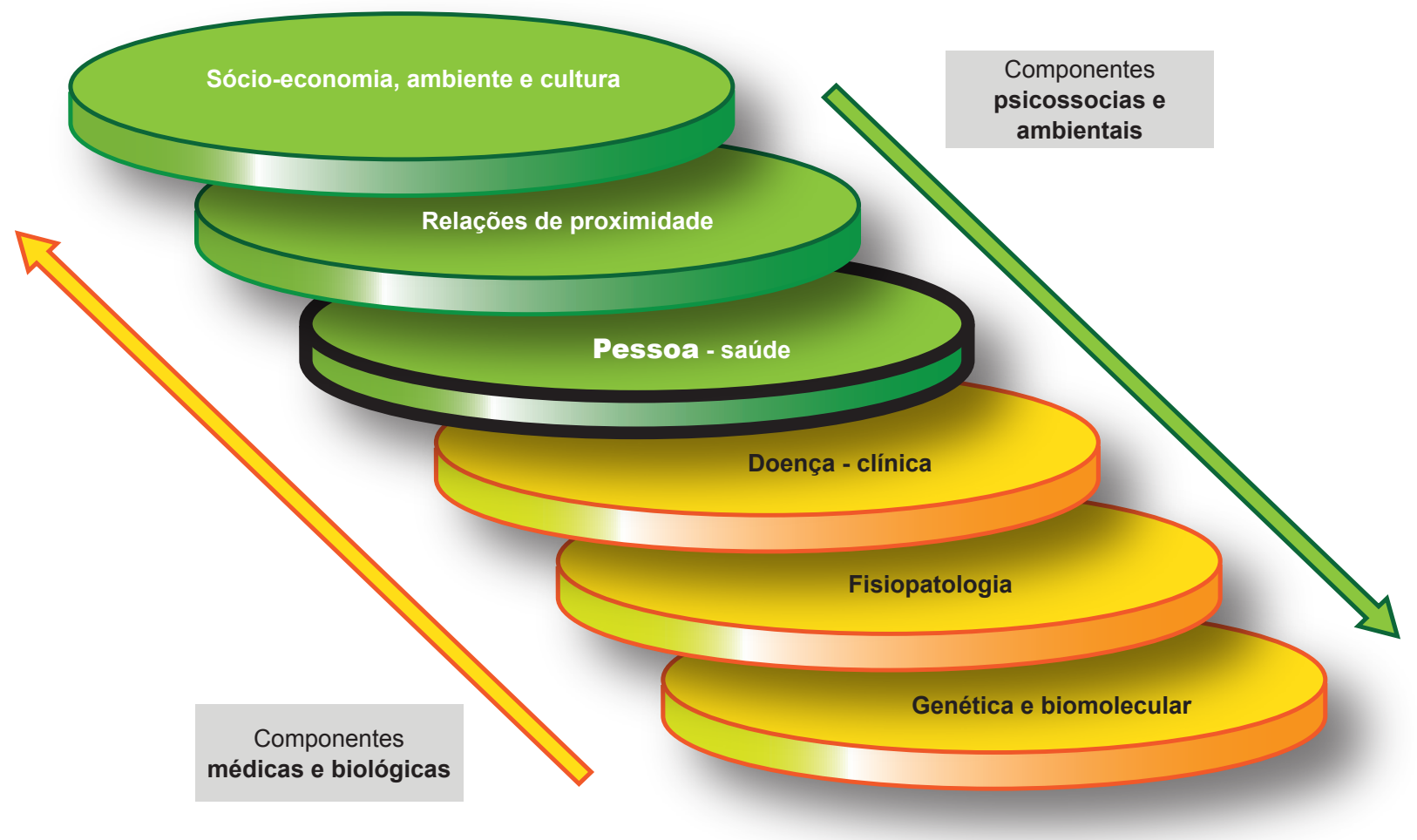

Figura 1 - Sistema adaptativo complexo: 'rede das redes' interativas a vários níveis. A assimetria entre as suas componentes biomédicas e psicossociais e a importância de postular uma centralidade - as pessoas - na 'rede das redes'. 
referentes a 40 morbilidades, publicado em 2012, ${ }^{10}$ estimou a prevalência da MM em 23,2\%. Observou-se também que a MM se inicia entre 10 e 15 anos antes nas pessoas que vivem em bairros pobres, quando comparadas com as que vivem nas melhores áreas residenciais, e que as perturbações de saúde mental acentuam-se quando se agravam os problemas de natureza física.

Concluíram os autores que estes dados" desafiam a abordagem dos cuidados centrados na doença (cada doença de per si), que atualmente informa a maior parte da prática, formação e investigação médicas".

Muito recentemente, a partir do primeiro inquérito nacional de saúde com observação física de, numa amostra probabilística representativa da população portuguesa de 4911 pessoas, com idades compreendidas entre os 25 e 74 anos, estimou-se em 38,2\% a prevalência da MM em Portugal. Observou-se também que esta prevalência aumenta com a idade e é maior nas mulheres e nas pessoas de níveis socioeconómicos mais baixos. ${ }^{11}$

O reconhecimento da importância da MM tem suscitado novas reflexões sobre a evolução do estado de saúde das pessoas, à medida que estas se desenvolvem e envelhecem.

Assim, para Joachim Sturmberg et a/ ${ }^{12}$ a MM é a expressão da resposta adaptativa complexa das redes interativas bio comportamentais e sócio ambientais de que fazemos parte, aos múltiplos estímulos que continuamente experimentamos. Esta resposta pode ser de molde a meIhorar a nossa capacidade de resiliência, ou pelo contrário pode resultar em processos adaptativos 'não saudáveis'. Outros autores subscrevem abordagens similares. ${ }^{13-15} \mathrm{O}$ reconhecimento desta 'rede de redes interativas' permite intervir simultânea e coordenadamente a vários níveis desse conjunto de redes.

Contudo, existe uma considerável assimetria nas bases de conhecimento e na prática dos cuidados de saúde, entre aquilo que diz respeito às redes de natureza biológica e aquelas que se situam a nível comportamental e social (Fig. 1). Esta assimetria está frequentemente associada a excessos no diagnóstico e terapêutica e suscita a necessidade de postular explicitamente uma centralidade (a pessoa) para a 'rede das redes'. ${ }^{16-20}$

De facto, o reforço da ideia da centralidade do cidadão no sistema de saúde é uma das consequências mais importantes desta transição da 'doença' para a 'pessoa' com múltiplos problemas de saúde. ${ }^{21-25}$

A noção da centralidade das pessoas na 'rede das redes' tem importantes implicações práticas (Fig. 2).

Dos múltiplos aspetos mencionados neste quadro destacam-se aqueles que são menos mencionados noutras secções deste trabalho:

Das doenças às pessoas: as boas praticas relativas a uma pessoa não são um somatório das boas práticas para cada uma das suas doenças

Há atualmente mais pessoas que vivem com morbilidade múltipla de que com uma só 'doença crónica'. Apesar
Boas práticas para a pessoa, não para cada uma das suas doenças Plano individual de cuidados - objetivos acordados com as pessoas Investimento na literacia e participação em saúde Decisão partilhada ao longo do percurso nos cuidados de saúde Decisões clínicas e a carga de trabalho por estar doente Partilha de experiências e grau de satisfação com serviços de saúde Reforço da capacidade de resolução dos cuidados de saúde primários

Figura 2 - Implicações práticas da centralidade do cidadão no sistema de saúde

disso, as normas de boas práticas continuam focadas na gestão de situações individualizadas. ${ }^{26}$ É necessário estabelecer orientações terapêuticas que tomem em consideração as interações entre as práticas que resultam das normas estabelecidas para cada doença de per si. ${ }^{27,28}$ Por outro lado, é importante reconhecer que as normas de boa prática clínica, nem sempre estão alinhadas com os desejos dos doentes. ${ }^{26}$

\section{Decisão partilhada ao longo do processo de cuida-} dos.

Há mais de 30 anos, Julian T. Hart, publicou "A New Kind of Doctor", ${ }^{29}$ obra baseada na sua experiência profissional naquilo que designou com a 'cocriação da saúde' com os seus doentes. Os progressos nesse sentido têm sido lentos, mas a decisão partilhada já faz parte da agenda da qualidade dos cuidados de saúde. ${ }^{30}$ São exemplo disso a importância hoje atribuída à 'negociação' com o doente na definição dos objetivos do seu plano individual de cuidados e as orientações no sentido de recorrer à decisão partilhada na gestão das situações de polifarmácia, particularmente nas pessoas com MM. ${ }^{31-33}$

\section{Carga de trabalho para o doente em consequência} dos cuidados de saúde

Tem-se observado que os progressos no diagnóstico e terapêutica, principalmente em pessoas com múltiplos problemas de saúde, têm sido acompanhados pelo agravamento da carga de trabalho que o doente tem de suportar como resultado dos cuidados de saúde. O diagnóstico e tratamento da doença podem ser quase tão disruptivos na vida da pessoas como a própria doença. É assim indispensável que as orientações e prescrições médicas, no decurso do processo de cuidados, tomem este aspeto em consideração, procurando minimizar aquela carga de trabalho. ${ }^{34-36}$

\section{Capacidade de resolução dos cuidados de saúde primários}

Bárbara Starfield, foi, de entre os investigadores em cuidados de saúde, talvez aquela que mais clara e precocemente chamou a atenção para o facto de o aumento de número de pessoas com MM ter sido acompanhado também pelo aumento da referenciação dos doentes para os 
cuidados hospitalares, ${ }^{37}$ que, quando precoces e desnecessárias, necessitam de ser contrariadas. Estas observações chamam a atenção para a necessidade de um aumento efetivo na capacidade de resolução dos cuidados de saúde primários. ${ }^{37,38}$

2. Desafio dois: desenvolvimento e vida ativa - retardar o início da morbilidade múltipla e atenuar a sua expressão.

Atrasar o início da MM e atenuar a sua expressão depende essencialmente de três aspetos:

- Desenvolvimento infantil: este faz-se por etapas críticas. Situações como o stress crónico na criança, nomeadamente quando ocorrem nessas etapas críticas para o desenvolvimento, podem ter efeitos duradouros. ${ }^{39,40}$

- Vida ativa: a promoção da saúde através de uma vida física, intelectual, afetiva e socialmente ativa é aqui de uma importância fundamental ${ }^{41-45}$ perspetivada ao longo do percurso de vida. ${ }^{46}$

- A vida ativa, o envelhecimento ativo, são essenciais para retardar o início da MM e atenuar os seus efeitos.

- Proteção da saúde, prevenção e gestão dos riscos de doença: esta faz-se hoje no âmbito de um amplo espectro de ações que vão desde os efeitos genéti$\cos ^{47}$ até aos das redes sociais, ${ }^{48}$ passando por muitas outros, que não é possível aqui mencionar. Neste contexto há que acentuar a relevância de uma gestão equilibrada entre o registo da "promoção da saúde" e o da "prevenção da doença".

\section{Desafio três: integração de cuidados centrada nas pessoas}

Resposta apropriada às pessoas com morbilidade múltipla
Ao acentuar-se o enfoque na MM como 'registo primário' nos cuidados de saúde, é importante recordar que, quando nas pessoas ocorre uma situação aguda ou de importância vital, o registo 'doença-índice e suas co-morbilidades' tende a sobrepor-se às outras preocupações, frequentemente de uma forma temporária.

A importância em dar uma resposta apropriada às pessoas com MM, está bem expressa no facto deste tema ser hoje considerado como uma prioridade global na investigação em cuidados de saúde, incluindo temáticas como as seguintes: que combinações de situações de saúde são mais problemáticas para as pessoas? Como aumentar os benefícios e reduzir os riscos no tratamento das pessoas com MM? Que consequências traz a MM para a organização dos cuidados de saúde?. ${ }^{49}$

Em relação a esta última questão parece haver, desde já, uma resposta clara: só com uma efetiva integração dos cuidados de saúde (e sua articulação com os cuidados sociais), superando a fragmentação que hoje se observa nos serviços de saúde, é possível começar a dar uma resposta apropriada à MM.

Existem múltiplas formas de integração de cuidados. ${ }^{50,51}$ Aqui, no entanto, prestar-se-á especial atenção, não tanto às integrações de carácter organizacional, mas àquela que se centra na gestão do percurso das pessoas através das organizações de saúde.

Gestão do percurso das pessoas através das organizações de saúde

O que interessa às pessoas que recorrem aos cuidados de saúde é serem capazes de percorrer os vários serviços de saúde de que necessitam, em tempo útil, com bons resultados no final de cada percurso. ${ }^{52-54}$ (Fig. 3).

Existe hoje um vasto acordo técnico sobre o facto de ser o 'plano individual de cuidados' (PIC) o instrumento essencial para uma efetiva gestão do percurso das pessoas

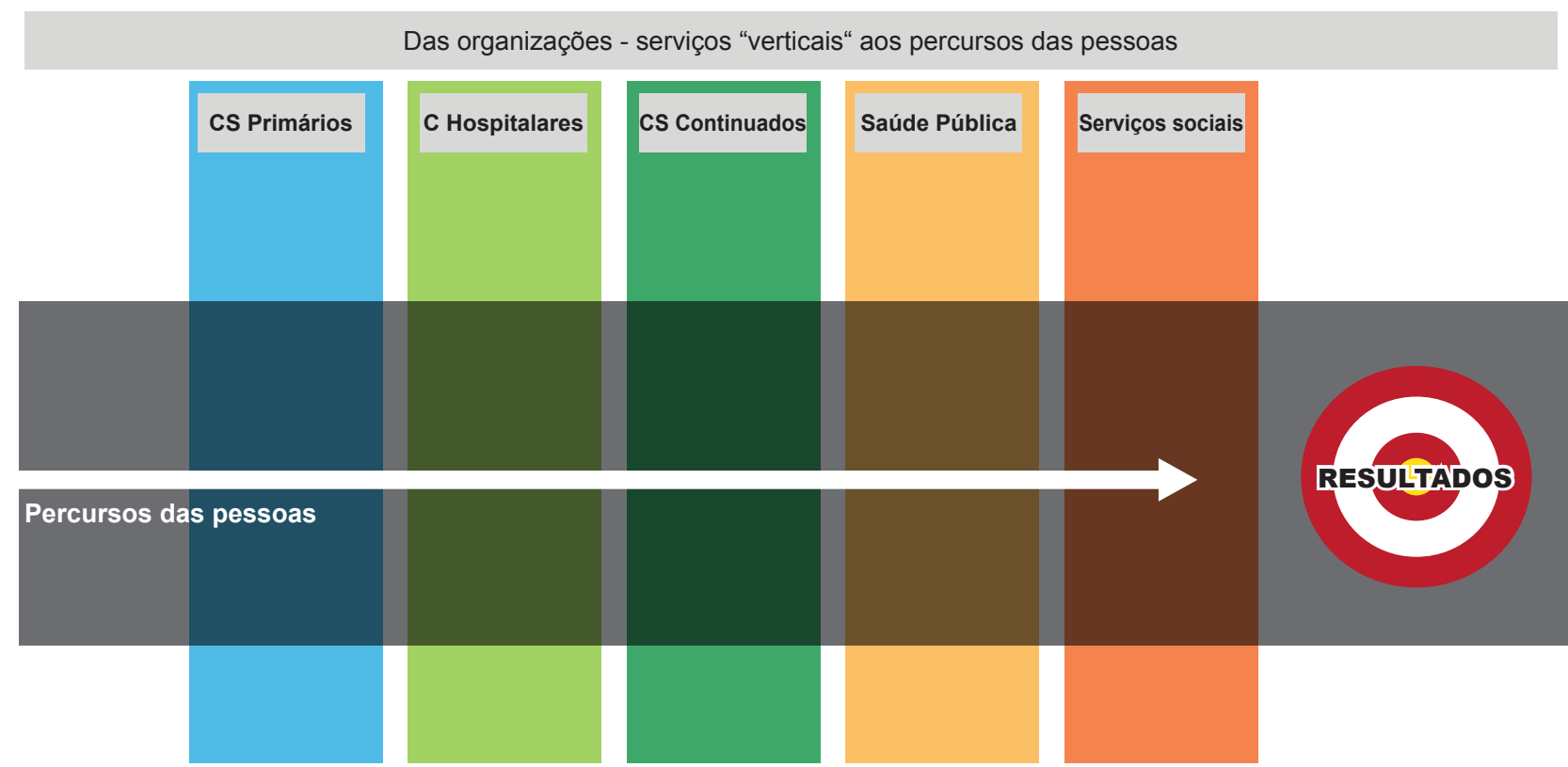

Figura 3 - Integração de cuidados centrada nos percursos das pessoa nos cuidados e saúde 
através dos serviços de que necessitam. ${ }^{55-57}$

Para fazer funcionar o PIC é indispensável melhorar o processo negocial na identificação dos objetivos do PIC, e na forma de os realizar, dar atenção às diferentes tipologias de pessoas com MM, estabelecer protocolos colaborativos com todos os profissionais cogestores do plano e proceder à monitorização e avaliação dos resultados da sua implementação. ${ }^{53}$

\section{PARTE II. Processos de mudança}

\section{Transformação um: princípios da gestão da mudan- ça em saúde}

A implementação de políticas de saúde transformadoras depende em grande parte do desenvolvimento das instituições de saúde e de uma ativação social para a mudança.

Um resumo acessível sobre as principais referências teóricas, relativas à gestão da mudança, pode encontrar-se em "Change Management in Health Care Literature", de $2014 .^{58}$

Nos programas de governo e nos discursos políticos da saúde, o foco tende a estar muito "no que fazer", e muito menos no "como fazer", ou seja, na efetiva implementação das políticas de saúde.

O reconhecimento deste facto, levou a Organização Mundial de Saúde na Europa, a iniciar, desde 2015, um projeto denominado "WHO Health Systems Transformation Initiative".59-61

Simplificando muito, podemos resumir o essencial do desafio da transformação na saúde, nos dois aspetos seguintes: (i) apostar na capacidade de promover mudanças adaptativas de proximidade, (ii) assegurar o enquadramento organizacional, de gestão e de decisão política necessários para que as mudanças de proximidade tenham lugar.

\section{Gestão de mudança adaptativa e de proximidade}

Num sistema complexo, a mudança é necessariamente adaptativa. Isto quer dizer que "no início do processo não há uma solução predefinida; ela é criada, emerge, no decurso do processo de mudança". ${ }^{61}$ Assim, a verdadeira mudança é sempre feita pelas equipas locais, próximas das pessoas. O papel das organizações é o de lhes proporcionar o enquadramento necessário, promovendo uma cultura organizacional amiga da mudança, alinhando a gestão dos recursos, da informação e da formação com aqueles processos de mudança. ${ }^{62,63}$

Reconhecer a complexidade, a incerteza e a não-linearidade associadas aos sistemas de saúde da atualidade, implica proporcionar um apoio determinado a líderes locais como empreendedores sociais/públicos, assertivos e flexíveis, "como disruptores criativos, capazes de instigar soluções inovadoras". ${ }^{64}$

Foi com base nestes princípios que foi idealizada a atual reforma dos cuidados de saúde primários em Portugal. ${ }^{65}$ As dificuldade da sua implementação e a ausência notória de movimentos transformativos comparáveis no setor hospitalar, está fortemente associada à inexistência do enquadramento organizacional, de gestão e de decisões políticas necessárias à sua materialização. ${ }^{66-68}$

Enquadramento organizacional, de gestão e de decisão política para as mudanças de proximidade

Um exemplo da natureza deste enquadramento está na iniciativa da Fundação Gulbenkian que resultou na publicação, em 2014, do trabalho "Um futuro para a saúde - todos temos um papel a desempenhar". ${ }^{69}$ As suas recomendações podem resumir-se da seguinte forma: os desafios (novo pacto para a saúde - do hospital e da doença para a pessoa e para a saúde), a participação (Conselho Nacional de Saúde, entre outras considerações), o conhecimento (o SNS-evidência) e o apoio à transformação ('fundo de transição').

O enquadramento da gestão adaptativa de proximidade é muito exigente:

Pede alinhamento no desenvolvimento organizacional e na gestão dos recurso com as mudanças locais

Requer uma gestão contínua da informação, do conhecimento e da inovação ${ }^{70-73}$;

Depende muito da qualidade da governança e do mo-

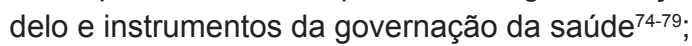

Não dispensa análise das agendas e comportamentos dos atores da saúde e as orientações estratégicas daí decorrentes. ${ }^{80-82}$

\section{Transformação dois: fatores indutores e facilitadores da mudança}

Estes são essencialmente as arquiteturas, processo e instrumentos colaborativos essenciais ao desenvolvimento institucional na ativação social no âmbito do sistema de saúde. Resumem-se na Fig. 4 os mais significativos desses fatores.

\section{Núcleos identitários e interfaces colaborativas}

Uma análise simples das organizações de saúde torna evidente o contraste entre a complexidade e o detalhe da sua gestão interna e a inexistência de diferenciação naquilo que diz respeito à sua interface colaborativa com outras organizações. O mesmo se pode dizer da maior parte das organizações profissionais. ${ }^{83-85}$ Tratar deste desequilíbrio entre os núcleos identitários e as interfaces colaborativas é muito relevante para a evolução dos sistemas de saúde.

\section{Da liderança heroica unipessoal para as lideranças} colaborativas

A prevalência de lideranças pós heroicas no sistema de saúde é outra condição necessária para as dinâmicas transformacionais que é necessário promover. Já há mais de uma década, alguém observou que "uma liderança serve melhor uma organização quando procura fazer sentido do mundo complexo que nos rodeia do que quando se limita a proporcionar respostas simples que prometem sucesso". ${ }^{86,87}$ 
Gestão do conhecimento e inteligência colaborativa: concertação efetiva entre setores, organizações e atores no sistema de saúde

O interesse pelos processos participativos no SNS tem-se reacendido ultimamente. ${ }^{88}$ Para isso pode vir a contribuir o facto de ser atualmente tecnicamente banal estratificar uma população alvo de acordo com as suas características e necessidades e direcionar a cada estrato aquela informação que é mais apropriada aos seus atributos e papel no sistema de saúde. $E$ também retroalimentar as bases de conhecimento a partir da forma como os diversos atores responderam à distribuição do conhecimento. Trata-se de fazer chegar a todos os atores da saúde, em ciclos contínuos, informação validada por processos facilmente escrutináveis, com o propósito de facilitar a colaboração entre eles na realização de objetivos comummente aceites. É aquilo que designamos por inteligência colaborativa. ${ }^{89,90}$

Da contratualização à distância para a contratualização colaborativa de proximidade

A contratualização do desempenho das unidades prestadoras de cuidados de saúde não dispensa uma forte base quantitativa - metas e indicadores de desempenho..$^{91,92}$
No entanto, a experiência internacional neste domínio tende a concluir que isso é insuficiente para conseguir o desempenho desejado. É de facto necessário acrescentar à base quantitativa habitual um processo qualitativo em termos de "planeamento conjunto do desempenho", entre financiadores, gestores e prestadores de cuidados, proporcionando-lhes um enquadramento colaborativo para a meIhoria desse desempenho. ${ }^{93,94}$

\section{Transformação três: direção estratégica do SNS -} principais domínios na gestão da mudança.

Necessitamos de um SNS que responda aos desafios do nosso tempo.

Esta transformação não se faz 'a frio', normativamente, num país alheio à qualidade das suas instituições e ao seu capital social e humano. Cabe a uma direção estratégica real, que o SNS precisará de instituir, fazer repercutir o conhecimento dos desafios da atualidade e a capacidade de gerir mudanças complexas sobre aqueles domínios que são essenciais à sua transformação - (i) financiamento (ii) gestão de infraestruturas e recursos (iii) desenvolvimento organizacional (iv) políticas para as profissões (Fig. 5).

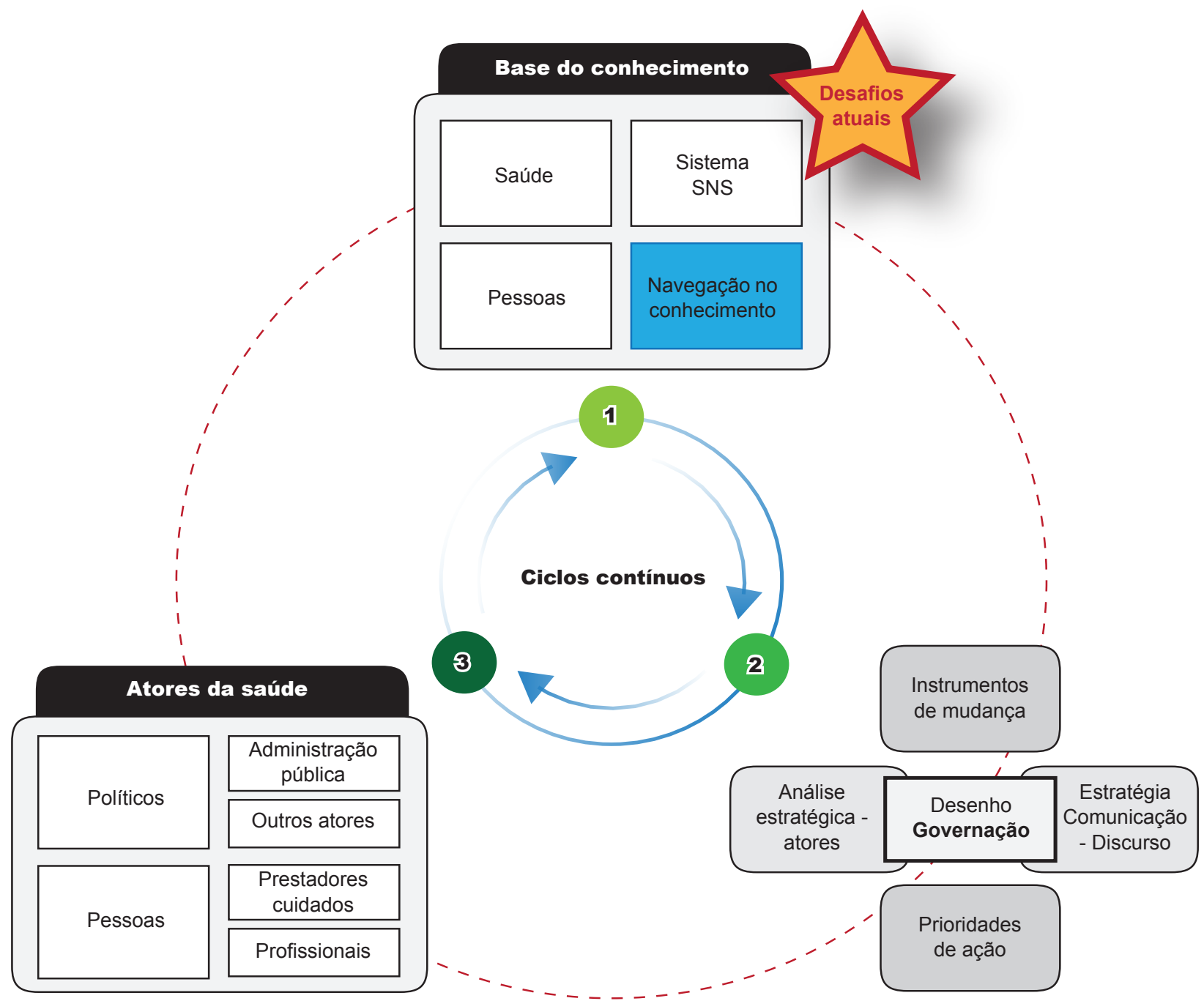

Figura 4 - Gestão do conhecimento e inteligência colaborativa em saúde 


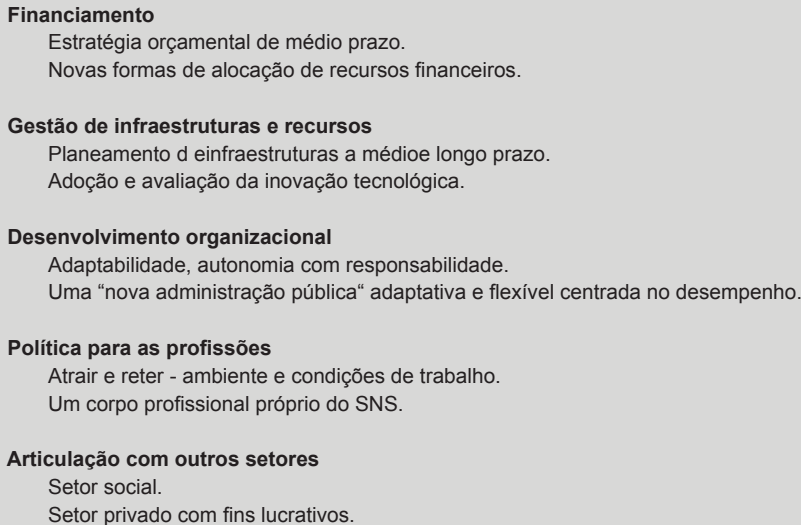

Figura 5 - Aspetos críticos da direção estratégica do SNS

\section{Financiamento e gestão dos recursos da saúde}

É necessário incluir na estratégia orçamental de médio prazo do país objetivos associados aos grandes desafios de saúde para os portugueses, acima referidos. ${ }^{95,96}$ Assim não sendo, será sempre pequena a possibilidade de meIhorar substancialmente a situação atual. Neste contexto é igualmente importante ser convincente quanto à capacidade em fazer evoluir o SNS de forma a melhor utilizar os recursos disponíveis. Desviar recursos financeiros públicos do SNS para evitar compromissos definitivos no investimento dos serviços públicos, cria um ciclo vicioso que conduz ao esvaziamento do SNS. ${ }^{97}$

\section{Gestão das infraestruturas e recursos}

É indispensável ter, e manter atualizado, um planeamento a longo prazo das infraestruturas do SNS. Isso requer um exercício prospetivo exigente sobre a evolução dos sistemas de saúde nas próximas décadas.

Os grandes desafios de saúde para os portugueses também dão indicações úteis sobre a necessidade de progredir na adoção e avaliação da inovação tecnológica na saúde (informação e comunicação, diagnóstico, tratamento incluindo o medicamento). ${ }^{98-102}$ Principalmente neste último domínio, conseguir melhorias substanciais implica uma melhor concertação entre os países da UE.

\section{Desenvolvimento organizacional}

Progressos no desenho organizacional do SNS (nos cuidados de saúde primários, continuados e hospitalares,

\section{Desafios atuais}

Desenvolvimento infantil e vida activa

Pessoas com morbilidade múltipla

Integração de cuidados centrada nas pessoas

Partilhar:

Comunicar com eficácia

\section{Transformação: Desenvolvimento institucional e ativação social}

\section{Princípios \\ - Mudança adaptativa de proximidade \\ - Enquadramento da ação local \\ - Informação, conhecimento e inovação \\ - Análise e orientações estratégicas \\ - Boa governança e governação}

\section{Fatores indutores e facilitadores da mudança}

- Arquiteturas, processos e intrumentos colaborativos

\section{Direção estratégica do SNS \\ - Financiamento \\ - Gestão de infraestruturas e recursos \\ - Desenvolvimento organizacional \\ - Política para as profissões \\ - Articulação com os setores social e privado com fins lucrativos}

Figura 6 - Desafios de saúde e gestão da mudança no SNS 
assim como na integração destes cuidados) requerem uma considerável evolução da administração pública da saúde..$^{103-105}$ Isto significa permitir, apoiar, acompanhar e continuamente avaliar a gestão adaptativa do desempenho das unidades do SNS, em vez de impor soluções centralizadas, preconcebidas e disfuncionais quando se trata de cuidados de saúde.

\section{Política para as profissões}

A especificidade das profissões da saúde é pouco compatível com simples aplicação de rotinas administrativas habituais na gestão dos recursos humanos.

Atrair e reter profissionais de saúde implica uma relação mais atenta, elaborada e flexível entre a gestão dos serviços a as exigências da prática profissional. Significa investir seriamente na qualidade do ambiente de trabalho e do exercício profissional (trabalho gratificante, aprendizagem contínua, participação na investigação e no ensino em saúde e cuidados de saúde), numa carreira orientada pelo reconhecimento do mérito e da excelência. ${ }^{106-109}$

Para isso muito contribuirá a institucionalização de um corpo profissional exclusivo do SNS.

\section{Articulação com o setores social e privado}

O SNS situa-se no centro de gravidade do sistema de saúde, que integra também um importante setor social e um setor privado com fins lucrativos em expansão. A forma como o SNS se articula com estes setores terá que constituir uma importante componente da direção estratégica do SNS.

\section{OBSERVAÇÕES FINAIS}

A grande entropia informativa do nosso tempo é agravada, em termos de política de saúde, por intermináveis descrições e medidas sobre a problemática da saúde, habitualmente nas suas vertentes mais imediatas.

Isso contribui para esbater o foco sobre o que é essencial, dificultando a mobilização de uma base social de apoio

\section{REFERENCIAS}

1. Stuckler D, Basu S. The body economic: why austerity kills. Recessions, budgets, battles and the politics of life and death. New York: Basic Books; 2013.

2. Sakellarides $C$, Castelo-Branco L, Barbosa P, Azevedo H. The impact of the financial crisis on the health system and health in Portugal - a case study. Copenhagen: WHO Regional Office for Europe on behalf of the European Observatory on Health Systems and Policies; 2014.

3. Santos MI. O doente com patologia múltipla em Medicina Geral e Familiar: comorbilidade de quatro doenças crónicas. Prémio Bial Menção honrosa. Porto: Bial; 2006.

4. Nicolau V, Nunes C, Escoval A. Estudo: prevalência da multimorbilidade em Portugal. In: Congresso Nacional de Saúde Pública. Lisboa: DGS; 2004.

5. Broeiro P, Ramos V, Barroso R. O mapa de problemas - um instrumento para lidar com a morbilidade múltipla. Rev Port Clin Geral. 2007;23:20915.

6. Broeiro P. Multimorbilidade e comorbilidade: duas perspectivas da mesma realidade. Rev Port Clin Geral. 2015;31:158-60.

7. Reis $\mathrm{S}$, Cardoso $\mathrm{S}$. Multimorbilidade em cuidados de saúde primários: o que há de novo? Rev Port Med Geral Fam. 2015;31:230-2.

8. Prazeres F, Santiago L. Prevalence of multimorbidity in the adult population attending primary care in Portugal: a cross- sectional study. suficiente para sustentar as transformações necessárias. ${ }^{110}$ Assegurar esse foco requer situá-lo naqueles que são, na atualidade, os grandes desafios de saúde para os portugueses e nas capacidades de gerir as transformações complexas que estes desafios requerem (Fig. 6).

É necessário investir mais recursos no SNS. Mas é igualmente importante transformar - descentralizar, integrar, inovar, democratizar - situar, sem excessos de retórica, as pessoas no centro do sistema de saúde. É preciso adotar um modelo de governação da saúde capaz de adotar estratégias de mudança centradas na gestão do conhecimento.

Essas transformações terão que ter em conta, simultaneamente, o património social e cultural do SNS, reforçando a sua marca identitária, ${ }^{111,112}$ e a forma como evoluem as sociedades contemporâneas. ${ }^{113-115}$ Assim será possível ir configurando um novo 'contrato social' para a saúde próprio dos nossos dias. ${ }^{1,90,116}$

\section{PROTEÇÃO DE PESSOAS E ANIMAIS}

Os autores declaram que os procedimentos seguidos estavam de acordo com os regulamentos estabelecidos pelos responsáveis da Comissão de Investigação Clínica e Ética e de acordo com a Declaração de Helsínquia da Associação Médica Mundial.

\section{CONFIDENCIALIDADE DOS DADOS}

Os autores declaram ter seguido os protocolos do seu centro de trabalho acerca da publicação de dados.

\section{CONFLITOS DE INTERESSE}

Os autores declaram não ter conflitos de interesses relacionados com o presente trabalho.

\section{FONTES DE FINANCIAMENTO}

Este trabalho não recebeu qualquer tipo de suporte financeiro de nenhuma entidade no domínio público ou privado.

BMJ Open. 2015;5:e009287.

9. Sakellarides C. Saúde em Portugal e na Europa - considerações a propósito do relatório "Health at a Glance: Europe 2015. Acta Med Port. 2019;32:7-10.

10. Barnett K, Mercer S, Norbury M, Watt G, Wyke S, Guthrie B. Epidemiology of Multimorbidity and implications for health care, research and medical education: a cross-sectional study. Lancet. 2012;380:37-43.

11. Quinaz-Romana G, Kislaya I, Salvador M, Cunha Gonçalves S, Nunes B, Dias C. Multimorbilidade em Portugal: Dados do primeiro inquérito nacional de saúde com exame físico. Acta Med Port. 2019;32:30-7.

12. Sturmberg, JP, Bennett JM, Martin CM, Picard M. 'Multimorbidity' as the manifestation of network disturbances. J Eval Clin Pract. 2017;23:199208.

13. Barabási A, Gulbahce N, Loscalzo J. Network medicine: a networkbased approach to human disease. Nat Rev Genet. 2011;12:56-68.

14. Bircher J, Hahn E. "Multimorbidity" as the manifestation of network disturbances. From nosology to the Meikirch model. J Eval Clin Pract. 2017;23:222-4.

15. Miles A, Asbridge J. Multimorbidity-a manifestation of network disturbances? How to investigate? How to treat? J Eval Clin Pract. 2017;23:193-8

16. Ziegelstein R. Personomics. J Am Med Assoc. 2015;175:888-9. 
17. Ziegelstein R. Perspectives in primary care: knowing the patient as a person in the precision medicine era. Ann Fam Med. 2018;16:4-5.

18. Damásio A. O livro da consciência: a construção do cérebro consciente. Lisboa: Editora Temas e Debates; 2010.

19. Zilioli S, Slatcher R, Ong A, Gruenewald TL. Purpose in life predicts allostatic load ten years later. J Psychosom Res. 2015;79:451-7.

20. Cohen R, Bavishi C, Rozanski A. Purpose in life and its relationship to all-cause mortality and cardiovascular events: a meta-analysis. Psychosom Med. 2016;78:122-33.

21. Vogt H, Hofmann B, Getz L. The new holism: P4 systems medicine and the medicalization of health and life itself. Med Health Care Philos. 2016;19:307-23.

22. Berwick D. Era 3 for medicine and health care. J Am Med Assoc. 2016;315:1329-30.

23. Miles A, Asbridge J. The chronic illness problem. The person-centered solution. Eur J Pers Cent Healthc. 2016;4:1-5.

24. Miles A, Asbridge J. From evidence-based to evidence-informed, from patient- focused to person-centered-The ongoing "energetics" of health and social care discourse as we approach the Third Era of Medicine. J Eval Clin Pract. 2017;23:3-4

25. Marcum J. Multimorbidity, P4 medicine and holism. J Eval Clin Pract. 2017;23:213-5

26. Millar E, Dowell A, Lawrenson R, Mangin D, Sarfati D. Clinical guidelines: what happens when people have multiple conditions? NZMJ. 2018;131:73-81.

27. Hughes L, McMurdo M, Guthrie B. Guidelines for people not for diseases: the challenges of applying UK clinical guidelines to people with multimorbidity. Age Ageing. 2013;42:62-9

28. Zamborini V, Silveira M, Pruski M, Teije A, Geleijn E, Leeden M, et al. Analysing interactions on combining multiple clinical guidelines. Art Intellig Med. 2017;81:78-93.

29. Hart JT. A new kind of doctor. London: The Marelin Press; 1998.

30. Elwyn G, Cochran N, Pignone M. Shared decision making the Importance of diagnosing preferences. JAMA Intern Med. 2017; 177:1239-40

31. Broeiro P, Maio I, Ramos R. Polifarmacoterapia: estratégias de racionalização. Rev Port Clin Geral. 2008;24:625-31.

32. Mair A, Fernandez-Llimos F, Alonso A, Harrison C, Hurding S, Kempen T, et al. Polypharmacy management by 2030 : a patient safety challenge. Coimbra: Simpathy Consortium; 2017

33. Scottish Government. Polypharmacy model of care group. Polypharmacy guidance, realistic prescribing. $3^{\text {rd }}$ edition. Scottish Government; 2018.

34. May C, Montori VM, Mair FS. We need minimally disruptive medicine. BMJ. 2009;339:b2803

35. Abu Dabrh A, Gallacher K, Boehmer K, Hargraves I, Mair F. Minimally disruptive medicine: the evidence and conceptual progress supporting a new era of healthcare. J R Coll Physicians Edinb. 2015;45:114-7.

36. Leppin A, Montori V, Gionfriddo M. Minimally disruptive medicine: a pragmatically comprehensive model for delivering care to patients with multiple chronic conditions. Healthcare. 2015;3:50-63.

37. Starfield B. Primary care/specialty care in the era of multimorbidity. Cancun: WONCA; 2010.

38. O'Malley A, Rich E. Measuring comprehensiveness of primary care: challenges and opportunities. J Gen Intern Med. 2015;30:568-75.

39. Conselho Nacional de Saúde. Gerações mais saudáveis: políticas públicas de promoção da saúde das crianças e jovens em Portugal. Lisboa: CNS, 2018.

40. Shonkoff J. The lifelong effect of early childhood adversity and toxic stress. Paediatrics. 2012;129:232-46.

41. Loureiro I, Miranda N, Pereira Miguel J. Promoção da saúde e desenvolvimento local em Portugal: refletir para agir. Rev Port Saúde Pública. 2013;31:23-31.

42. Hibbard J, Gilburt H. Supporting people to manage their health - an introduction to patient activation. London: The Kings Fund; 2014.

43. Wit L, Fenenga C, Giammarchi C, di Furia L, Hutter I, de Winter A, et al. Community based activities improving critical health literacy: Systematic review and meta-synthesis of qualitative evidence. BMC Public Health. 2018;18:40.

44. Pedro AR. Literacia em Saúde: da gestão da informação à decisão inteligente. Tese para a obtenção do grau de Doutor em Saúde Pública. Lisboa: Escola Nacional de Saúde Pública. Universidade Nova de Lisboa; 2018. p.175-94.

45. Silva Costa A, Arriaga M, Veloso Mendes R, Miranda D, Barbosa P, Sakellarides $\mathrm{C}$, et al. A strategy for the promotion of health literacy in Portugal, centered around the life-course approach: the importance of digital tools. Port J Public Health. 2019;1-5
46. Burton-Jeangros C, Cullari S, Sacker A, Blane D, editores. A life course perspective on health trajectories and transitions. Berlin: Springer Open 2015.

47. Hollands G, French D, Griffin S, Prevost A, Sutton S, King S, et al. The impact of communicating genetic risks of disease on risk- reducing health behaviour: systematic review with meta-analysis. BMJ. 2016;352:i1102.

48. Levenson JC, Shensa A, Sidani JE, Colditz JB, Primack BA. The association between social media use and sleep disturbance among young adults. Prev Med. 2016;85:36-41.

49. Academy of Medical Sciences. Multimorbidity: a priority for global research. London: AMS; 2018.

50. Santana $R$, Marques AP, coordenadores. Integração vertical de cuidados de saúde. Lisboa: Escola Nacional de Saúde Pública, Obras Avulsas 09; 2016.

51. World Health Organization. Strengthening people-centered health systems in the WHO European: a framework for action in integrated health services delivery. In: WHO Regional Committee for Europe, $66^{\text {th }}$ session. Copenhagen: WHO Regional Office for Europe; 2016.

52. Williams $D$, Hutton $H$, Ryan $G$. Creating value in healthcare: the need for innovative solutions. Healthc Pap. 2017;16:47-51.

53. Ministério da Saúde. SNS + Proximidade. Mudança centrada nas pessoas. Lisboa: MS; 2017.

54. Stokes J, Struckmann V, Kristensen S, Fuchs S, Ginneken E, Tsiachristas A, et al. Towards incentivizing integration: a typology of payments for integrated care. Health Policy. 2018:122:963-9.

55. National Clinical Guideline Centre. Multimorbidity: clinical assessment and management. Multimorbidity: assessment, prioritization and management of care for people with commonly occurring multimorbidity. NICE Guideline, methods, evidence and recommendations Programme. London: Division of Health Systems and Public Health; 2016

56. ICARE4EU. Strategies to support patient-centred care for multimorbidity patients. Brussels: ICARE4EU; 2016.

57. Glaser J. From electronic health record to electronic health plan. Hospital \& Health Networks; 2015.

58. Antwi M, Mruganka K. Change Management in Health Care Literature. The Monieson Centre for Business Research in Health Care, Queen's School of Business, 2014.

59. World Health Organization. Regional Office for Europe. Health system transformation: making it happen. Expert meeting Madrid, Spain, 17-18 December 2015. Copenhagen: Regional Office for Europe; 2016

60. World Health Organization. Regional Office for Europe. Leading health systems transformation to the next level. Expert meeting Durham, United Kingdom, 12-13 July 2017. Copenhagen: WHO Regional Office for Europe; 2018

61. Kluge $H$, Hunter D, Bengoa R, Jakubowski E. Leapfrogging the elephant: making health systems transformation happen faster. Eurohealth. 2018;24:32- 6

62. Olivie D. Radical pragmatism and medical leadership. BMJ. 2019;365:14339.

63. Bohmer R. The hard work of health care transformation. N Engl J Med. 2016;375:709-11.

64. Blake N. New era for health services will focus on systems and creativity-an essay. BMJ. 2018;362:k2605.

65. Ministério da saúde. Grupo consultivo para a reforma dos cuidados de saúde primários - Acontecimento extraordinário: SNS proximidade com qualidade: relatório do grupo consultivo para a reforma dos cuidados de saúde primários. Lisboa : MS; 2009.

66. Firmino-Machado J, Magalhães I, Rodrigues J, Ramos V, Baptista S, Vilas Boas B. Governação de saúde e gestão de proximidade: necessidade de autonomia de gestão nos agrupamentos de centros de saúde. Acta Med Port. 2017;30:431-3.

67. Associação Nacional de Unidade de Saúde Familiares. $7 \times 7$ medidas Um novo ciclo dos cuidados de saúde primários (2015-2018). Lisboa: ANUSF; 2015

68. Barbosa P. Descentralização na Administração Pública e na organização administrativa da saúde. In: Barbosa P. (2018) Descentralização na organização e gestão do Serviço Nacional de Saúde e dos cuidados de saúde primários em Portugal. Tese para Obtenção do Grau de Doutor em Saúde Pública - Política, gestão e administração de saúde. Lisboa: Escola Nacional de Saúde Pública, Universidade Nova de Lisboa; 2018. p. 53-70.

69. Fundação Calouste Gulbenkian. Um Futuro para a Saúde - Todos temos um papel a desempenhar. Lisboa: FCG; 2014.

70. Kitson A, Brook A, Harvey G, Jordan Z, Marshall R, O'Shea R, et al. Using complexity and network concepts to inform healthcare knowledge translation. Int J Health Policy Manag. 2018;7:231-43. 
71. Langlois EV, Montekio VB, Young T, Song K, Alcalde-Rabanal J, Tran N. Enhancing evidence informed policymaking in complex health systems: lessons from multisite collaborative approaches. Health Res Policy Syst. 2016;14:20

72. Wutzke S, Benton M, Verma R. Towards the implementation of large scale innovations in complex health care systems: views of managers and frontline personnel. BMC Res Notes. 2016;9:3-5.

73. Sakellarides C. A Lisbon agenda on health innovation. Eur J Public Health. 2008;18:102-3.

74. Sakellarides C. Stewardship. In: Marinker M, editor. Constructive conversations about health and values. Oxford: Radcliffe Publishing; 2006.

75. Swanson D, Bhadwal S, editores. Creating adaptive policies : a guide for policy making in an uncertain world. Ottawa: International Institute for Sustainable Development. The Energy and Resources Institute. International Development Research Centre; 2009.

76. Ruger J. Shared health governance. Am J Bioeth. 2011;11:32-45

77. Braithwaite J, Churruca K, Ellis LA, Long J, Clay-Williams R, Damen $\mathrm{N}$, et al. Complexity science in healthcare - aspirations, approaches, applications and accomplishments: a white paper. Australian Institute of Health Innovation. Sydney: Macquarie University; 2017.

78. Ansell C, Sorenson E, Torfing J. Improving policy implementation through collaborative policymaking. Policy \& Politics. 2017;45:467-86.

79. Santos Al. Gestão da mudança na saúde: do planeamento à governança em saúde. Um contributo para a governança local em saúde. Lisboa: Escola Nacional de Saúde Pública, Universidade Nova de Lisboa; 2018.

80. Mintzberg H, Lampel J. Reflexão sobre o processo estratégico. Revista Portuguesa de Gestão. 2000:24-34.

81. Varvasovsky Z, Brugha R. How to do (or not to do) a stakeholder analysis. Health Policy Plan. 2000;15:338-45.

82. Braithwaite J. Changing how we think about healthcare improvement. BMJ. 2018;361:k2014.

83. Rummier GA, Blache AP. Improving performance: how to manage the white space on the organizational chart. San Francisco: Jossey-Bass; 1991.

84. Gittell JH, Godfrey M, Thistlethwaite J. Interprofessional collaborative practice and relational coordination: improving healthcare through relationships. J Interprof Care. 2013;27:210-3.

85. Korner M, Butof S, Muller C, Zimmermann L, Becker S, Bengel J. Interprofessional teamwork and team interventions in chronic care: a systematic review. J Interprof Care. 2016; 30:15-28.

86. Timmins $\mathrm{N}$. The practice of system leadership: being comfortable with chaos. London: The King's Fund; 2015. [consultado 2019 jun 19]. Disponível em: https://www.kingsfund.org. uk/sites/default/files/field/ field_publication_file/System-leadership Kings-Fund-May-2015.pdf.

87. West M, Eckert R, Steward K, Pasmore B. Developing collective leadership for health care. London: The King's Fund; 2014. [consultado 2019 jun 19]. Disponível em: https://www. kingsfund.org.uk/sites/default/ files/field/field_publication_file/develop-ing-collective-leadershipkingsfund-may14.pdf.

88. Crisóstomo S, Santos M. Participação pública na decisão em saúde. O que temos aprendido? Rev Port Farmacotert. 2018;10:118-20.

89. Wilson HJ, Daugherty PR. Collaborative intelligence: humans and Al are joining forces. Harvard Business Review. 2018:3-11.

90. Sakellarides C, Escoval A, Barbosa P, Santos AI, Pedro AR, Miranda, $D$. Health systems at the stage of complexity: The need for collaborative intelligence In: Health Care and Government Policy [online]. Évora. [consultado 2019 jun 19]. Disponível em: http://books.openedition.org/ cidehus/8291.

91. Escoval A. O processo de contratualização na saúde em Portugal (1996-2005). Rev Port Saúde Pública. 2010;9:7-24.

92. Administração Central do Sistema de Saúde IP. Termos de referência para a contratualização de cuidados de saúde primários no SNS para 2019. Lisboa: ACSS; 2018.

93. Klasa K, Greer SL, van Ginneken H. Strategic purchasing in practice: comparing ten European countries. Health Policy. 2018;122:457-72.

94. Collins B. Payments and contracting for integrated care. The false promise of the self-improving health system. London: The King's Fund; 2019.

95. Chalersworth A, Bloor K. 70 years of NHS funding: how do we know how much is enough? BMJ. 2018;361:k2373.

96. Trigo Pereira $P$, Cabral $R$, Morais LT, Vicente JA. Uma estratégia orçamental sustentável para Portugal. Coimbra: Almedina; 2018.

97. Conselho Nacional de Saúde. Fluxos financeiros nos SNS. Lisboa: CNS, 2017

98. Thomson S, Schang L, Chernew ME. Value-based cost sharing in The United States and elsewhere can increase patients use of high-value goods and services. Health Affairs. 2013;32:1-9.

99. Aranda da Silva J. Vinte e cinco anos de evolução do INFARMED, I.P.: os atuais desafios. Rev Port Farmacoter. 2017;9:113-7.

100. Sakellarides C, Pedro AR, Mendes MS. Knowledge-centered health innovation: the case for citizen health information systems. In: Kickbusch I, editor. Policy innovation for health. New York: Springer Science. Business Media, LLC; 2009.

101. Laranjo L. Person-centered care and health information technology in Portugal. Implications for chronic care and health quality improvement. Tese de doutoramento em medicina e epidemiologia. Lisboa: Faculdade de Medicina de Lisboa; 2015.

102. Sawesi S, Rashrash M, Phalakornkule K, Carpenter JS, Jones JF. The impact of information technology on patient engagement and health behavior change: a systematic review of the literature. JMIR Med Inform. 2016;4:1 -24.

103. Madureira C, Asensio M. O desafio de mobilizar e requalificar a Administração Pública. In: Paes Mamede R, Adão e Silva P, coordenadores. O estado da nação e as políticas públicas 2019: menos reformas, melhores políticas. Lisboa: IPPS-ISCTE; 2019. p. 68-71.

104. The Evidence Centre. Complex adaptive systems. The Health Foundation; 2010.

105. Kreindler SA, Dowd DA, Dana Star N, Gottschalk T. Silos and social identity: the social identity approach as a framework for understanding and overcoming divisions in health care. Milbank Q. 2012;90:347-74.

106. Barbazza E, Langins M, Kluge H, Tello J. Health workforce governance: processes, tools and actors towards a competent workforce for integrated health services delivery. Health Policy. 2015;119:1645-54.

107. Correia T, Dussault G, Pontes C. The impact of the financial crisis on human resources for health policies in three southern-Europe countries. Health Policy. 2015;119:1600-5.

108. Barriball L, Bremner J, Buchan J, Craveiro I, Dieleman M, Dix O, et al. Recruitment and retention of the health workforce in Europe. Final Report. Brussels: Eur Commission; 2015.

109. Correia T, Dussault G, Gomes I, Augusto M, Temido M, Nunes P. Recursos humanos na saúde: o que se sabe e o que falta saber. In Relatório da Primavera: 68-107. Lisboa: Observatório Português dos Sistemas de Saúde; 2018.

110. Ham C. The 10-years plan: transformation holds the key to sustainability. The NHS 10-year plan/transformation holds the key to sustainability. The King's Fund; 2018. [consultado em 2018 dez 10]. Disponível em. https:// www. kingsfund.org.uk/blog/2018/07/nhs-10-year-plan-transformationholds- key-sustainability.

111. Fundação para a Saúde - SNS. Serviço Nacional de Saúde: para uma conversação construtiva. Lisboa: Diário de Bordo, 2014.

112. Fundação para a Saúde - SNS. Serviço Nacional de Saúde: breve interpretação e linhas para a sua transformação. Lisboa: Fundação para a Saúde - SNS; 2019.

113. Simões J. Retrato político da saúde - dependência do percurso e inovação em saúde: da ideologia ao desempenho. Coimbra: Almedina; 2004.

114. Merkel W. Past, present and future of democracy. Policy Review. Brussels: Eur Comission; 2019.

115. De Leeuw E, Clavier C, Breton E. Health policy - why research it and how: health political science. Health Res Policy Syst. 2014;12:55.

116. Sakellarides C. Novo contrato social da saúde: incluir as pessoas. Lisboa: Saúde \& Sociedade; 2009. 